\title{
Le micro-usinage par laser et ses applications
}

\author{
John LOPEZ, Président du Club Laser et Procédés, \\ Ingénieur de recherche CELIA / ALPHANOV \\ john.lopez@alphanov.com
}

La miniaturisation s'invite aujourd'hui dans tous les secteurs industriels majeurs. En effet, la plupart des procédés de fabrication développés pour le macroscopique sont transposés à l'échelle microscopique. Généralement, on parle de micro-usinage lorsque les dimensions, les tolérances ou la zone affectée sont à l'échelle du micron. On distingue les procédés d'usinage par enlèvement de matière (perçage, découpe, gravure, marquage en surface ou en volume), les procédés de fabrication additive (rechargement, stéréo lithographie, frittage, polymérisation à deux photons) et enfin les procédés d'assemblage (soudage, brasage). Nous discuterons ci-après les potentialités des lasers industriels travaillant par enlèvement de matière, notamment pour le micro-usinage des matériaux métalliques. Nous présenterons également quelques applications impliquant les polymères ou les verres.

es possibilités offertes en termes d'usinage par une même machine laser et la qualité d'usinage atteinte permettent de s'affranchir de nombreuses étapes d'usinage, de pré- ou de post-traitement, ce qui conduit ainsi à diminuer le temps et le coût de fabrication d'un produit donné. Par ailleurs, l'absence de contact entre l'outil et la pièce autorise l'usinage de pièces très fragiles telles que des feuillards ou des couches minces. En revanche, le contrôle de la zone affectée thermiquement (ZAT) est en général un point critique qui nécessite d'adapter le choix de la source laser et les paramètres opératoires de manière à maîtriser le processus d'interaction laser-matière ainsi que les effets secondaires induits.

\section{Laser continu ou impulsionnel?}

L'interaction entre un rayonnement laser infrarouge (IR) ou visible avec un métal donne lieu à un processus thermique : absorption de l'énergie par les électrons, chauffage, diffusion thermique, fusion, vaporisation. Avec un rayonnement laser continu, la découpe s'effectue généralement en utilisant un gaz d'assistance, injecté sous pression dans la saignée, de façon coaxiale par rapport au faisceau. Ce gaz a une action mécanique et vient chasser le bain fondu et ainsi accélérer la découpe. II peut également avoir une action chimique et inhiber une éventuelle oxydation de la dépouille. Ce procédé est utilisé par exemple pour la découpe de stents en acier inox par laser à fibre pour les applications en angioplastie. II existe de nombreuses applications similaires dans les domaines de la micromécanique et du médical.

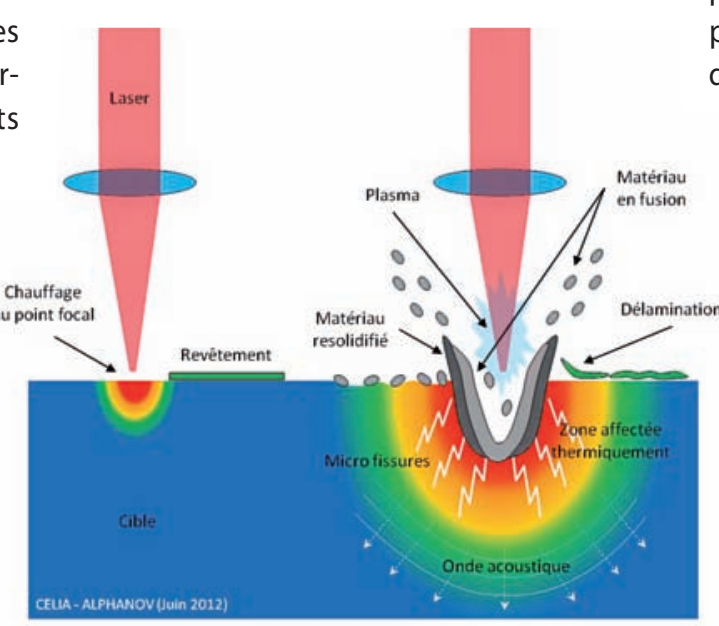

Processus thermique

Figure 1. Processus thermique lors d'un usinage par un laser à impulsions longues (>> 1 ps).
Ces lasers continus peuvent être également utilisés pour réaliser un marquage sans gravure.

Àl'opposé, l'utilisation d'un laser impulsionnel permet d'accroître l'intensité sur cible et de favoriser ainsi une découpe par fusion/vaporisation, voire directement une sublimation du métal. L'utilisation du gaz devient alors facultative. La profondeur usinée à chaque impulsion est parfaitement contrôlée, l'usinage peut donc être débouchant ou non (borgne). Le régime impulsionnel convient à la fois pour des applications de découpe fine et pour des opérations de gravure, de marquage ou encore de perçage.

\section{Les lasers à impulsions 《longues»}

Pour des impulsions dites longues ( $>1 \mathrm{ps}$ ), le débit matière et l'étendue de la zone affectée thermiquement s'accroît avec la durée d'impulsion. Cela s'explique simplement dans la mesure où, plus l'impulsion est longue, plus la chaleur diffuse et affecte un grand volume de matière. Par exemple, il est possible de percer $4 \mathrm{~mm}$ de laiton en une seule et unique impulsion, de durée $1 \mathrm{~ms}$ et d'énergie plusieurs dizaines de 
joules. Ainsi, avec un processus thermique, le débit matière est élevé mais les effets collatéraux sont étendus (bavures, endommagement et dénaturation de la cible, délamination), de telle sorte que pour certaines applications, il est nécessaire de brider la puissance délivrée sur la cible pour limiter les effets thermiques et avoir une qualité de surface exploitable (figure 1). De plus, la finition de la pièce requiert en général un post-traitement d'ébavurage, de polissage ou de nettoyage. C'est le cas par exemple lors de la gravure de motif en bas-relief sur des matrices d'emboutissage, des électrodes EDM ou encore des moules d'injection plastique, qui s'effectue aujourd'hui par laser nanoseconde (à solide ou à fibre), et qui nécessite fréquemment une opération de finition par microbillage ou par polissage électrochimique pour avoir un état de surface acceptable (soit une rugosité moyenne $(R a)$ inférieure à $0,3 \mu \mathrm{m})$. Pour ces opérations de gravure, le débit matière est typiquement de l'ordre de 5 à $10 \mathrm{~mm}^{3} / \mathrm{mn}$. Une autre application des lasers impulsionnels nanosecondes concerne le perçage de silicium pour les cellules photovoltaïques à technologie EWT (emitter wrap through) ou MWT (metallization wrap through). Le perçage permet de reporter le contact électrique en face arrière et de limiter ainsi les zones inactives sur la face avant. Dans ce cas précis, le perçage s'effectue à la volée à une cadence de plusieurs milliers de trous à la seconde (diamètre 100 à $200 \mu$ m environ).

Pour une pièce donnée, il est possible de prédire les zones susceptibles affectées par la chaleur en observant le tracé de la pièce. Ainsi, les zones à faible rayon de courbure ou les fines bandes de matière vont concentrer l'accumulation de chaleur et exacerber localement les effets thermiques. Les bavures, déformations ou autres effets indésirables liés à la chaleur vont donc préférentiellement apparaître sur ces zones-là. II est donc important d'optimiser la dynamique du déplacement du spot laser sur la cible de manière à diminuer localement le temps d'exposition de la matière au faisceau. Ainsi, pour des travaux de découpe fine ou de gravure, il sera bien souvent préférable d'accroître la vitesse de balayage du faisceau sur la cible, notamment par l'utilisation de têtes galvanométriques (dites "scanners») ou de déflections acousto-optiques, et de multiplier le nombre de passages lasers pour atteindre la profondeur usinée souhaitée. La bonne gestion des effets thermiques sur la cible est donc primordiale.

\section{Micro-usiner les matériaux sensibles à la chaleur}

La zone affectée thermiquement est un critère important lorsqu'il s'agit d'usiner des matériaux sensibles à la chaleur tels que des composites à matrices céramiques (CMC) ou organiques (CMO). En effet, le laser est aujourd'hui utilisé pour le perçage, la gravure ou encore le décapage de tels matériaux. Un choix judicieux de la source laser et des paramètres opératoires permet de minimiser les effets de délamination et l'endommagement de la matrice. Une application émergente par exemple est le micro perçage de bords d'attaque de voilure, de soufflante ou d'arrière-corps de moteur pour le secteur aéronautique. La multiperforation améliore à la fois l'aérodynamisme et l'émission de bruits des pièces traitées. Pour des trous millimétriques avec un faible niveau de perforation, les lasers de perçage conventionnels (durée d'impulsion milliseconde) suffisent; en revanche pour des trous de plus faible diamètre ou pour atteindre une forte densité de trous, il est préférable d'utiliser des lasers de gravure (durée d'impulsion nanoseconde), pour lesquels la quantité d'énergie requise (dose) pour percer un trou équivalent peut être dixà vingt fois plus faible, et l'endommagement matière bien inférieur (figure 2).

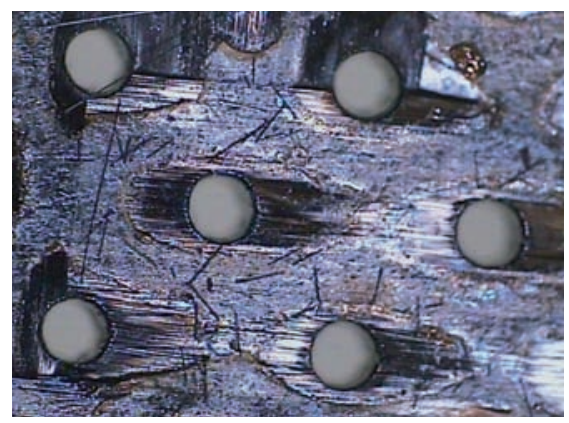

Figure 2. Multiperforation de trous de faible diamètre dans une plaque de carbone époxy.

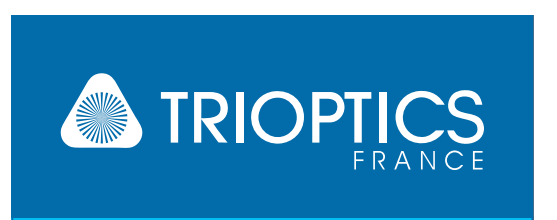

UN UNIVERS DE PRÉCISION

- Solutions complètes en interférométrie

et vibrométrie laser

- Mesure ultra-précise de déplacements, d'angles et de spectres vibratoires

- Résolution 0,1 nm

- Têtes de mesure compactes.

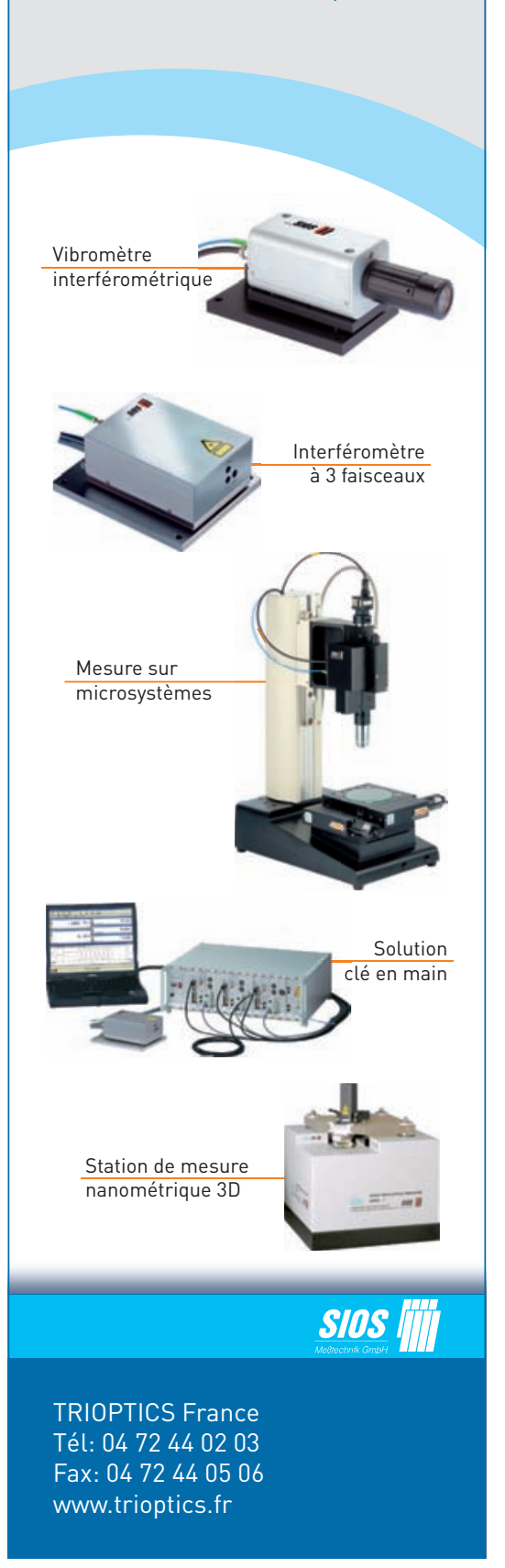




\section{Cas particulier des polymères}

Pour le micro-usinage des polymères, la problématique est toute autre car il est alors primordial d'éviter l'échauffement du matériau pour en conserver son intégrité, sa forme et ses propriétés. Un rayonnement laser ultraviolet (UV) interagissant avec un polymère absorbant donnera un processus de décomposition photochimique. Contrairement au rayonnement IR, l'énergie de chaque photon UV est suffisante pour casser une liaison covalente. Au-delà d'un certain nombre de liaisons ainsi brisées, il y a expulsion de la matière. Plus le polymère absorbe, moins ily aura d'effets thermiques. Ce processus, dit de photoablation, est mis à profit pour des applications phares telles que la chirurgie de la cornée en ophtalmologie (LASIK) ou le perçage de vias sur des circuits électroniques, mais aussi pour des applications plus confidentielles comme la réalisation de trous calibrés sur des capteurs de gaz portables ou encore le perçage de cathéters en polymère. L'absorption des matériaux augmente généralement lorsque la longueur d'onde diminue, ainsi certains polymères qui n'absorbent pas à $355 \mathrm{~nm}$ (laserYAG triplé) absorberont bien à $266 \mathrm{~nm}$ (laser YAG quadruplé). L'absorption d'un polymère commercial donné dépend principalement de sa formulation propre. Ainsi, le PI et le PEN s'usinent bien à $355 \mathrm{~nm}$. À l'inverse pour le PET, le PU ou le PS, I'ablation à $355 \mathrm{~nm}$ donnera des effets thermiques et il faudra usiner à $266 \mathrm{~nm}$ pour atteindre une meilleure qualité d'usinage. Pour les polyoléfines ou le PMMA, il sera préférable d'utiliser une longueur d'onde plus courte (193 nm) pour une qualité d'usinage optimale. La présence d'additif (1 à $2 \%$ ), tel que du noir de carbone par exemple, peut modifier sensiblement l'absorption d'un polymère donné et le rendre plus absorbant.

\section{Ablation sélective}

L'ablation laser est un phénomèneà seuil, c'est-à-dire qu'elle n'apparaît qu'au-delà d'un cer- tain seuil énergétique. En outre, la différence de seuil d'ablation entre les différents matériaux est bien plus marquée dans I'UV que dans I'IR. Ainsi, le seuil d'ablation des polymères dans le domaine spectral UV est dix à vingt fois plus faible que celui des métaux, des verres, de la silice ou du silicium; il est donc possible de réaliser l'ablation sélective d'une couche de polymère sans affecter le substrat en verre sous-jacent par exemple. Ce procédé est utilisé pour le perçage de vias en micro-électronique ou encore pour le dénudage de câbles électriques ou de fibres optiques.

Par ailleurs, les matériaux en couche mince (épaisseur $<<1 \mu \mathrm{m}$ ) ont un seuil d'ablation bien inférieur à celui du matériau brut (typiquement d'un facteur 10). Il est donc possible d'effectuer l'ablation sélective d'une couche mince (métallique ou oxyde) sur un substrat métallique ou un verre, voire même un polymère. Ce procédé d'ablation sélective est utilisé aujourd'hui dans le domaine du photovoltaïque pour les opérations de patterning sur les cellules solaires basées sur la technologie couche mince (CIGS, Cd-Te, organique...). Bien entendu, la qualité du résultat et la robustesse du procédé pour une application donnée dépendent fortement de la longueur d'onde (IR, visible ou UV), de la durée d'impulsion (ns, ps ou fs) et du profil spatial du faisceau (gaussien ou « top hat »). Pour les opérations de pat- terning sur les écrans des smartphones et de tablettes, sur les écrans plats (TFT et LCD), sur les smart windows (opaques ou transparents à la demande) ou sur l'électronique organique (OLED et AMOLED), I'ablation sélective est obtenue par insolation laser UV (excimère).

\section{L'intérêt des impulsions ultracourtes}

Pour des durées d'impulsions inférieures à $10 \mathrm{ps} \mathrm{l'homme} \mathrm{de} \mathrm{l'art} \mathrm{parle} \mathrm{d'im-}$ pulsions courtes, voire ultracourtes. Une telle durée d'impulsion est inférieure au temps caractéristique de diffusion de la chaleur pour la plupart des matériaux (figure 3). La matière est expulsée avant que la chaleur ne diffuse en dehors du volume irradié. La matière ablatée emporte donc avec elle l'excès de chaleur et les effets thermiques résiduels sur la cible sont considérablement réduits par rapport à des impulsions longues (figure 4). La transition entre le régime thermique et le régime ultrabref se situe entre 1 à 50 ps selon les matériaux.

La qualité d'usinage est optimale (faible endommagement matière, précision extrême), en revanche la quantité de matière ablatée par impulsion est faible comparativement à une impulsion dite longue. Les lasers ultracourts industriels actuels permettent de compenser efficacement ce défaut grâce à un taux de répétition pouvant atteindre plusieurs $\mathrm{MHz}$ et une puissance moyenne de plusieurs dizaines de watt, voire supérieure à 100 watts. Par exemple, des travaux récents montrent qu'il est possible de réaliser la gravure de cylindre d'embossage en cuivre avec un débit matière de $20 \mathrm{~mm}^{3} / \mathrm{min}$ avec ce type de source. En termes de qualité de surface, il est possible de descendre à une rugosité moyenne (Ra) de 0,2 voire 0,1 micron sur des alliages

Figure 3. Phénomènes mis en jeu en fonction de la longueur d'onde du laser utilisé pour l'usinage. 


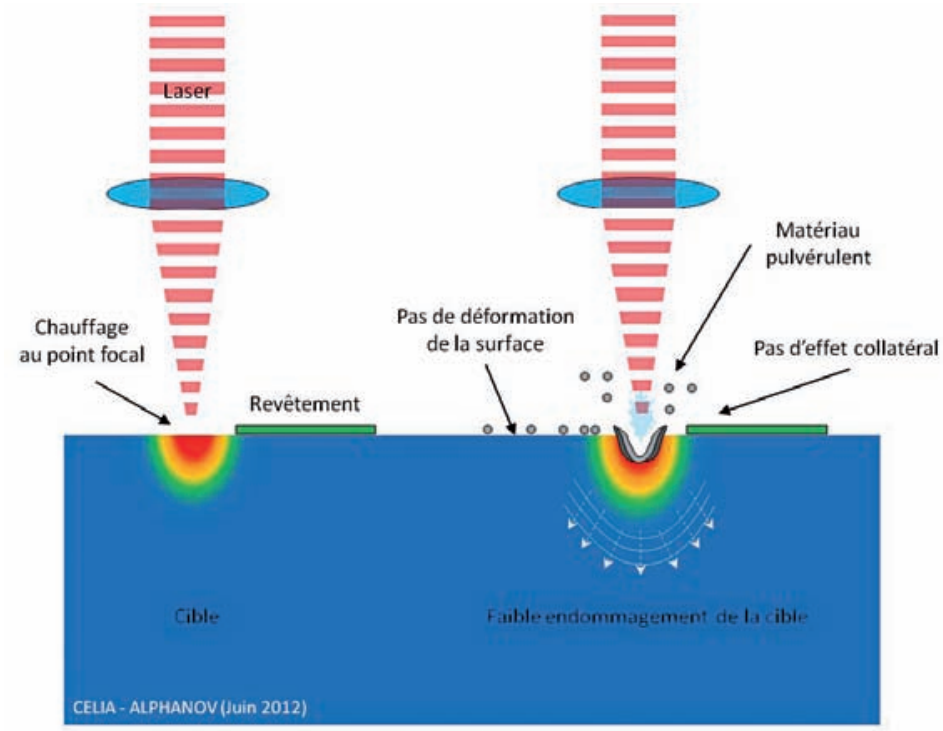

Processus ultrabref

Figure 4. L'utilisation de lasers à impulsions ultracourtes permet de réduire les effets thermiques observés dans la matière.

métalliques. Bien évidemment, gérer un tel taux de répétition et une telle puissance moyenne demande un effort particulier sur la dynamique du scanner et la synchronisation entre le scanner et le tir laser, afin d'éviter les éventuels problèmes d'accumulation thermique dans la cible et d'assurer une excellente qualité d'usinage (figure 5). Par ailleurs, compte tenu des fortes intensités mises en jeu $\left(10^{12}\right.$ à $10^{14} \mathrm{~W} / \mathrm{cm}^{2}$ sur cible), l'énergie requise pour amorcer l'ablation est bien inférieure à celle nécessaire avec une impulsion longue. Ainsi, quelques $\mu \mathrm{J}$ peuvent suffirent pour bon nombre d'applications de micro usinage.

\section{Repousser les limites du possible}

Cette technologie ouvre la voie à de nouvelles possibilités d'usinage jusqu'alors irréalisables. Elle permet d'usiner avec précision aussi bien les matériaux durs (diamants, nitrure de bore, carbures, saphir, alliages métalliques) que les matériaux sensibles à la chaleur (composites, polymères, tissus vivants, couches minces). Plusieurs applications industrielles ont pu voir le jour depuis 2 à 3 ans, telles que la découpe de pièces diverses pour la micromécanique, la découpe de stents en polymère biodégradable (PLLA) pour le médical (figure 6), la découpe de céra- mique PZT pour l'aéronautique, la texturation de cylindre sur moteur automobile, ou encore la gravure de pièces en saphir ou céramique pour l'horlogerie.

\section{Texturer et fonctionnaliser les surfaces}

Ces lasers ultracourts permettent également un vaste panel d'applications en termes de texturation ou de fonctionnalisation de surfaces métalliques pour améliorer les propriétés aérodynamiques, esthétiques ou tribologiques, ou encore les rendre super hydrophobes (lotus-like) ou super absorbantes à la lumière (black silicium, black gold). La texturation peut être obtenue par gravure directe, par interférométrie ou par un effet de polarisation (figure 7). II est possible de créer simultanément deux niveaux de textura-

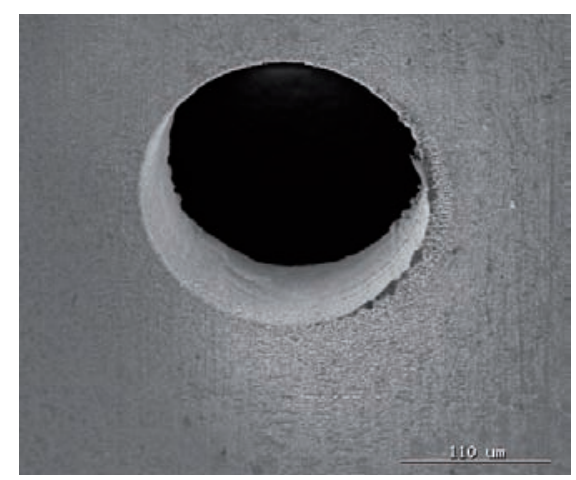

Figure 5. Micro-perçage sur acier par lasers femtoseconde. Les impulsions ultracourtes évitent les problèmes $d$ 'accumulation thermique dans la cible et assurent une excellente qualité d'usinage.

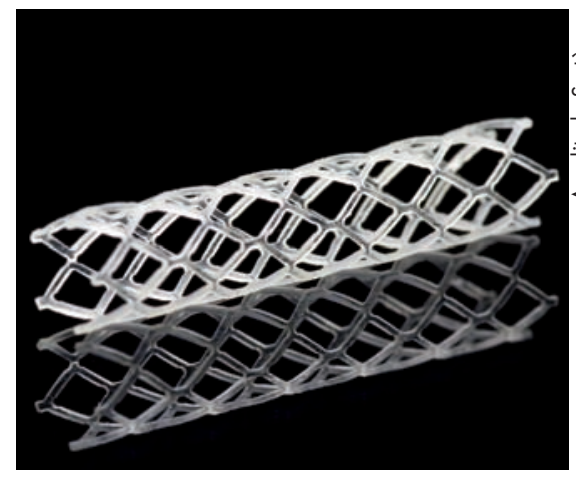

Figure 6. Stent en polymère bio-résorbable découpé par laser femtoseconde.

tion, I'un à l'échelle micrométrique et l'autre à l'échelle nanométrique.

\section{Modifier la matière dans son épaisseur}

Une autre caractéristique des impulsions ultracourtes est la possibilité d'avoir une absorption non-linéaire locale dans des matériaux a priori transparents (diélectriques, polymères). Cela est uniquement possible car l'intensité lumineuse

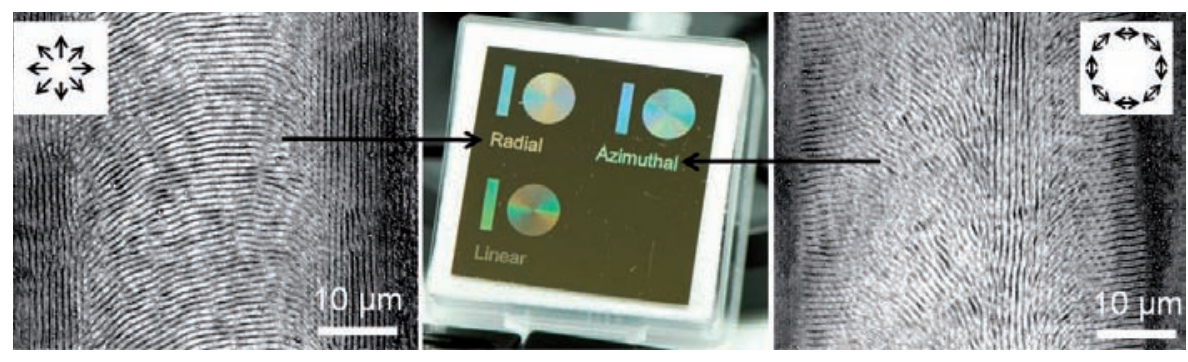


est très élevée et il existe une forte probabilité au point focal que plusieurs photons cumulent leur énergie et rendent localement le matériau absorbant, on parle alors d'absorption multiphotonique. Ce processus non-linéaire confère la possibilité unique de modifier la matière dans l'épaisseur du matériau, sans altérer la surface, de manière à générer localement une variation de densité ou d'indice de réfraction (biréfringente ou non) (figure 8). II n'y a pas fissuration dans la matière. Ainsi, on peut créer un marquage en volume (par exemple un code DataMatrix) ou un guide d'onde. La découpe du capot cornéen par laser femtoseconde pour la chirurgie réfractive (dit FemtoLASIK) utilise un procédé similaire; dans ce cas, chaque impulsion laser femtoseconde produit une bulle de gaz dans le collagène de la cornée et la prédécoupe du capot est possible en créant un plan de bulles sous la surface.

\section{Découper le verre}

La découpe de verre est un sujet d'actualité, en particulier pour la découpe des écrans de smartphones ou de tablettes. Les techniques de découpes par scie diamantée ou par ablation laser génèrent une forte quantité de poussières et ne sont pas toujours adaptées (faible rayon de courbure impossible par voie mécanique et découpe par ablation laser trop lente); par ailleurs le champ de coupe est rugueux et microfissuré. Par une mise en forme de faisceau spécifique, il est possible de combiner la réalisation d'une entaille de quelques microns en surface avec la réalisation de micro filaments (produits par absorption non linéaire) prolongeant l'entaille dans l'épaisseur du verre et permettant un clivage ultérieur. Ce nouveau procédé permet de s'affranchir des poussières d'ablation, d'améliorer la qualité de coupe et surtout d'atteindre des vitesses 10 à 20 fois supérieures à celles obtenues par ablation laser seule.

\section{Réaliser des structures multifonctionnelles 3D dans la silice}

Par ailleurs, des travaux récents ont montré que la densification locale produite au point focal du laserfemtoseconde dans de la silice accélère d'un facteur 60 environ la gravure chimique par HF (5\%).

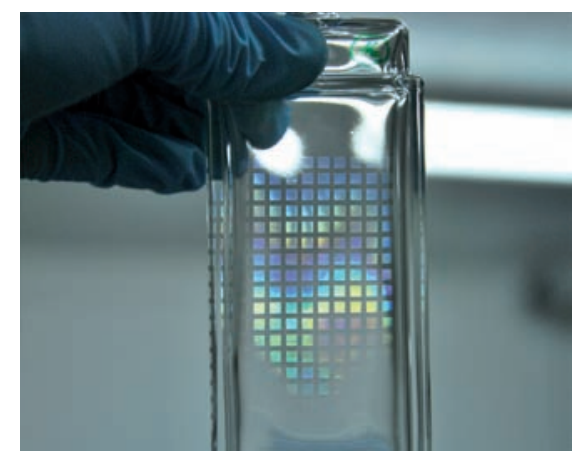

Figure 8. Marquage intravolume diffractif dans un flacon de parfum en verre.

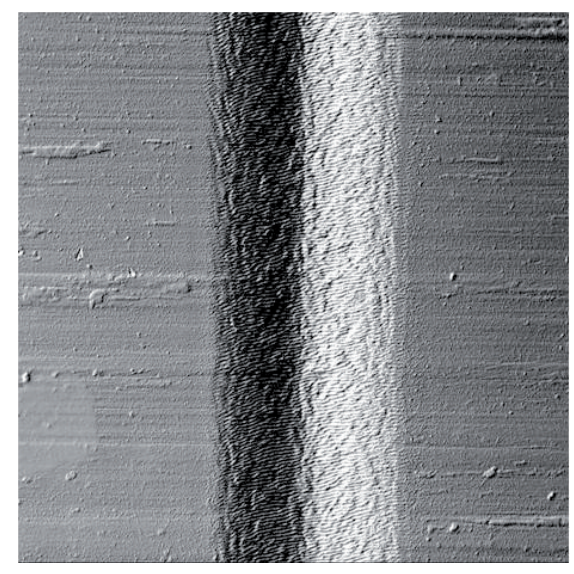

Figure 9. Gravure dans du molybdène par laser femtoseconde à $2 \mathrm{MHz}$.

Il est donc possible d'insoler selon un motif défini dans la masse ou en surface et de réaliser ensuite une gravure chimique sélective uniquement du volume de matière exposé au laser. Ce procédé de gravure chimique assistée par laser est particulièrement prometteur pour la réalisation de lab-on-a-chip pour le médical (analyse de sang, analyse ADN, etc.) et la biologie (analyse de toxicité, culture cellulaire, etc.). Il permet aussi de réaliser des composants multifonctionnels monolithiques combinant des fonctions optiques, mécaniques et de manipulation de fluides.

\section{Combiner qualité d'usinage et productivité}

Les développements récents sur les technologies pico et femtoseconde permettent aujourd'hui de disposer de sources industrielles de forte puissance moyenne et à très haute cadence ( $\mathrm{kHz}$ voire $\mathrm{MHz}$ ), lesquelles arrivent même à surpasser en productivité les autres technologies laser pour des applications de prédécoupe de verre ou silicium (scribing), de gravure (figure 9) ou de fonctionnalisation de surface (implants médicaux, pièces de moteur automobile), d'ablation sélective de couches minces ou encore de marquage intra-volume anti-contrefaçon sur flacons de vaccin ou de parfum. Les chiffres sont éloquents puisqu'un code DataMatrix $16 \times 16$ cellules se grave aujourd'hui en moins de $100 \mathrm{~ms}$, ce qui rend le procédé compatible avec les cadences requises pour le conditionnement de produits pharmaceutiques notamment. En revanche, il est à noter que, contrairement aux impulsions longues pour lesquelles le débit matière augmente avec la durée d'impulsion, le débit matière diminue généralement en passant du régime femtoseconde au régime picoseconde ; cette différence d'efficacité entre les régimes pico et femto dépend des propriétés du matériau. Cette différence est faible dans le cas du cuivre mais importante dans le cas de l'aluminium, du molybdène ou du silicium par exemple.

\section{Ce qu'il faut en retenir...}

Le micro-usinage laser est une solution technologique en plein essor qui allie efficacement flexibilité et performances. II confère la possibilité d'usiner un grand nombre de matériaux avec une parfaite maîtrise de l'endommagement matière et une grande précision. Le micro-usinage laser adresse aujourd'hui de nombreux secteurs applicatifs (médical, microfluidique, micromécanique, aéronautique, horlogerie, optique, électronique, photovoltaïque, automobile, e-mobilité, etc.) et se révèle particulièrement productif pour des applications de gravure ou texturation de surface ou intra-volume. Les développements récents en termes de technologie laser permettent aujourd'hui d'usiner un matériau avec un minimum d'effets thermiques et de préserver ainsi les fonctionnalités de la pièce. Si le choix de la source laser est primordial, la qualité du résultat et le temps d'usinage dépendent fortement du procédé d'usinage, de la mise en forme du faisceau et du contrôle de procédé. 